Émeline Sessi Pélagie Asséd ${ }^{1}$

Fortuné Akomian AzıHOU ${ }^{1}$

Aristide Cossi AdOMOU ${ }^{2}$

Madjidou OUMOROU ${ }^{3}$

Brice SINSIN ${ }^{1}$

${ }^{1}$ Université d'Abomey-Calavi Faculté des sciences agronomiques Laboratoire d'écologie appliquée 01 BP 526, Cotonou

Bénin

2 Université d'Abomey-Calavi Faculté des sciences et techniques Département de biologie végétale Herbier national du Bénin 01 BP 4521, Cotonou

Bénin

3 Université de Parakou

École nationale supérieure d'aménagement

et de gestion des aires protégées Laboratoire d'écologie appliquée 01 BP 526, Cotonou

Bénin

\section{Effet du relief sur la régénération des espèces ligneuses en zone soudanienne du Bénin}

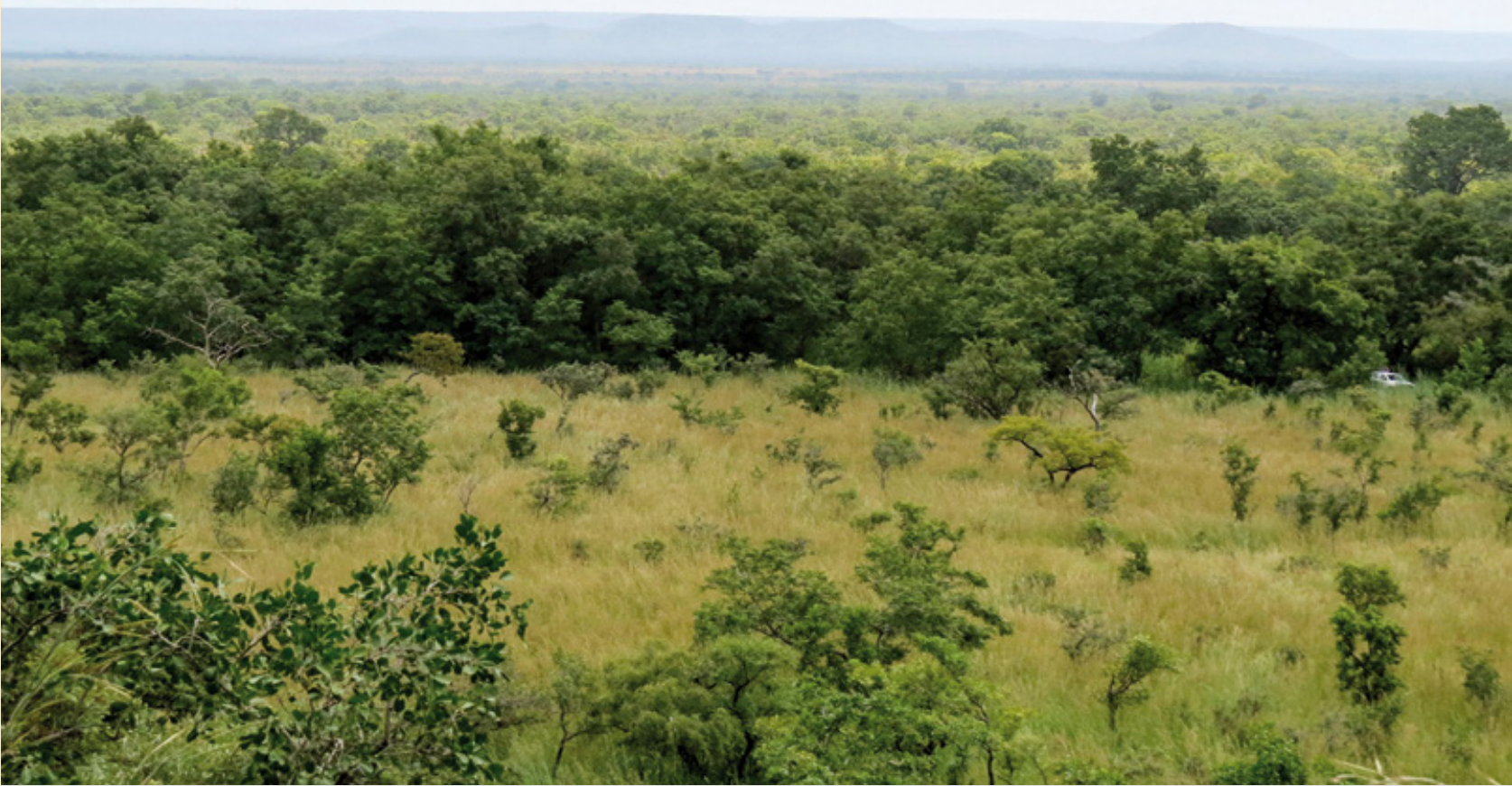

Photo 1.

Vue panoramique de la végétation de la Réserve de biosphère de la Pendjari : une mosaïque de savanes et de forêts.

Photo E. Assédé. 


\section{RÉSUMÉ}

\section{EFFET DU RELIEF SUR LA RÉGÉNÉRATION DES ESPÈCES LIGNEUSES EN ZONE SOUDANIENNE DU BÉNIN}

La régénération des espèces ligneuses est un des domaines d'intérêt majeurs des écologues dans le processus de renouvellement des écosystèmes. La présente étude visait, d'une part, à analyser le potentiel de régénération des unités de végétation en zone soudanienne au Bénin et, d'autre part, à renseigner ses variations en fonction du relief. Au total, 615 placettes carrées de $25 \mathrm{~m}^{2}$ ont été matérialisées à l'intérieur de treize unités de végétation dans la Réserve de Biosphère de la Pendjari. La régénération a été inventoriée en relevant dans chaque placette le nombre d'individus par espèce. Le modèle log-linéaire associé à la loi de Poisson a permis de modéliser la densité de régénération et le nombre d'espèces en régénération selon quatre types de relief. Le potentiel de régénération des unités de végétation de la Réserve de biosphère de la Pendjari reflète bien la composition floristique de la végétation en place. La régression de Poisson montre que le relief a un effet significatif sur la densité de régénération et le nombre d'espèces en régénération. Le potentiel de régénération au sein des plaines d'inondation et bordures de cours d'eau est faible par rapport à celui des collines et plateaux. Les densités de régénération observées sur les bordures de cours d'eau, les plaines d'inondation et les collines sont respectivement de $1,01,0,67$ et 2,1 fois supérieures à celles observées sur les plateaux. Le nombre d'espèces en régénération augmente des plateaux vers les collines, mais diminue des plateaux vers les plaines d'inondation et les bordures de cours d'eau. Les conditions topographiques influencent l'établissement des espèces ligneuses en zone soudanienne.

Mots-clés : potentiel de régénération, communauté végétale, régression de Poisson, Pendjari, Bénin.

\section{ABSTRACT}

\section{EFFECTS OF THE RELIEF ON THE REGENERATION OF WOODY SPECIES IN BENIN'S SUDANIAN ZONE}

The regeneration of woody species is of major interest to ecologists in connection with ecosystem renewal. This study aimed (i) to analyse the regeneration potential of vegetation units in Benin's Sudanian zone, (ii) to document the variations in regeneration potential according to the relief. A total of 615 survey plots $25 \mathrm{~m}^{2}$ in area were marked out in 13 vegetation units in the Pendjari Biosphere Reserve. An inventory of regeneration was made by counting the number of individuals per species in each plot. We used the log-linear model with a Poisson regression to model regeneration density and the number of regenerating species for four types of relief. The regeneration potential of vegetation units in the Pendjari Biosphere Reserve closely reflects the species composition of the plant cover. The Poisson regression shows that the relief has a significant effect on regeneration density and on the number of regenerating species. Regeneration potential in floodplains and along rivers is significantly lower than in hilly and plateau areas. Compared to plateau areas (index 1 ), the indices for observed regeneration density along rivers, in floodplains and in hilly areas are $1.01,0.67$ and 2.1 respectively. The number of regenerating species increases from plateau to hilly areas, but drops from plateau areas to floodplains and riversides. Topographic conditions thus influence the establishment of woody species in Sudanian zones.

Keywords: regeneration potential, plant community, Poisson regression, Pendjari, Benin.

\section{EFECTO DEL RELIEVE EN LA REGENERACIÓN DE ESPECIES LEÑOSAS EN LA ZONA SUDANESA DE BENÍN}

La regeneración de especies leñosas es uno de los principales centros de interés de los ecólogos dentro del proceso de renovación de los ecosistemas. Este estudio tenía por objetivo: (a) analizar el potencial de regeneración de las unidades de vegetación en la zona de clima sudanés de Benín e (b) informar de las variaciones según el relieve. Se establecieron 615 parcelas cuadradas de $25 \mathrm{~m}^{2}$ dentro de trece unidades de vegetación en la Reserva de Biosfera de Pendjari. Se realizó un inventario de la regeneración registrando en cada parcela el número de individuos por especie. Utilizando la regresión loglineal de Poisson se pudo modelizar la densidad de regeneración y el número de especies en regeneración según cuatro tipos de relieve. El potencial de regeneración de las unidades de vegetación de la Reserva de Biosfera de Pendjari refleja bien la composición florística de la vegetación existente. La regresión de Poisson muestra que el relieve tiene un efecto significativo en la densidad de regeneración y en el número de especies en regeneración. El potencial de regeneración de planicies inundables y riberas de cursos de agua es bajo con respecto al de colinas y mesetas. Las densidades de regeneración observadas en riberas de cursos de agua, planicies de inundación y colinas fueron, respectivamente, 1.01, 0.67 y 2.1 superiores a las observadas en las mesetas. El número de especies en regeneración aumenta de las mesetas hacia las colinas y disminuye de las mesetas hacia las planicies inundables y riberas de cursos de agua. Las condiciones topográficas influyen en el establecimiento de especies leñosas en la zona de clima sudanés.

Palabras clave: potencial de regeneración, comunidad vegetal, regresión de Poisson, Pendjari, Benín. 


\section{Introduction}

La régénération naturelle des communautés végétales est un processus complexe qui est contrôlé par plusieurs facteurs d'ordre biologique, écologique, démographique et historique (Bucci et Borghetti, 1997). La régénération des espèces ligneuses dans le processus de renouvellement des écosystèmes est un des domaines d'intérêt majeurs des écologues. Dans la dynamique des successions végétales, les recrûs de plants contribuent au maintien de la diversité spécifique des ligneux et la structure de la végétation future se dessine à partir des populations existantes de juvéniles (Maingi et Marsh, 2006).

En Afrique de l'Ouest, l'étude de la régénération dans le contexte de la dynamique des peuplements végétaux a connu un essor au cours de la dernière décennie (Sinsin et al., 2004 ; Gaoué et Ticktin, 2007, 2009 ; Koulibaly et al., 2010). Elle révèle que le modèle de distribution des arbres et de leur régénération est affecté par de nombreux facteurs biotiques et abiotiques et leurs interactions. Le potentiel de régénération est favorisé non seulement par une densité optimale d'individus semenciers mais aussi par des relations de facilitation (Sinsin et al., 2004 ; Biaou et al., 2011). Les travaux conduits sur la démographie de la composante juvénile (Ouédraogo et al., 2006, 2009 ; Avocevou-Ayisso et al., 2009), les mécanismes de régénération (Ky-Dembele et al., 2007) et l'impact de l'anthropisation sur la structure de la végétation (Bondé et al., 2013 ; Avocevou-Ayisso et al., 2009 ; Schumann et al., 2011 ; Nacoulma et al., 2011) ont également mis en évidence le rôle déterminant du sol dans l'établissement et la régénération des ligneux.

Les études ayant traité de l'effet de la topographie sur la régénération concernent surtout les forêts tempérées de montagne (Coop et al., 2010 ; Robert, 2003). Elles montrent l'effet négatif de l'élévation et de la pente sur la régénération forestière, et l'effet positif des trouées sur la structure en diamètre et en hauteur des populations (Coop et al., 2010 ; Robert, 2003). Mais dans le contexte du suivi des végétations en zone soudanienne, particulièrement exposées à la déforestation (Mama et al., 2014), la question de la relation entre le relief et le potentiel de régénération (le nombre d'espèces en régénération et la densité de régénération) reste posée.

Un potentiel de régénération est nécessaire pour qu'un écosystème dispose d'une bonne dynamique végétale. Même si le stock en semences, le pouvoir germinatif de chaque graine et les modes de dispersion restent des facteurs déterminants de ce potentiel, les conditions édaphiques n'en sont pas moins primordiales pour la régénération (Ouédraogo et al., 2009). La Réserve de biosphère de la Pendjari (RBP), dont les objectifs prennent en compte la préservation des espèces et des écosystèmes, doit accorder une grande importance à ce potentiel de régénération. L'objectif de cette étude est d'évaluer le potentiel de régénération des unités de végétation de la RBP et le rôle du relief dans l'expression de ce potentiel. Nous avons retenu les deux questions de recherche suivantes : quel est le potentiel de régénération des unités de végétation identifiées dans la RBP et comment ce potentiel de régénération varie-t-il suivant les conditions du relief?

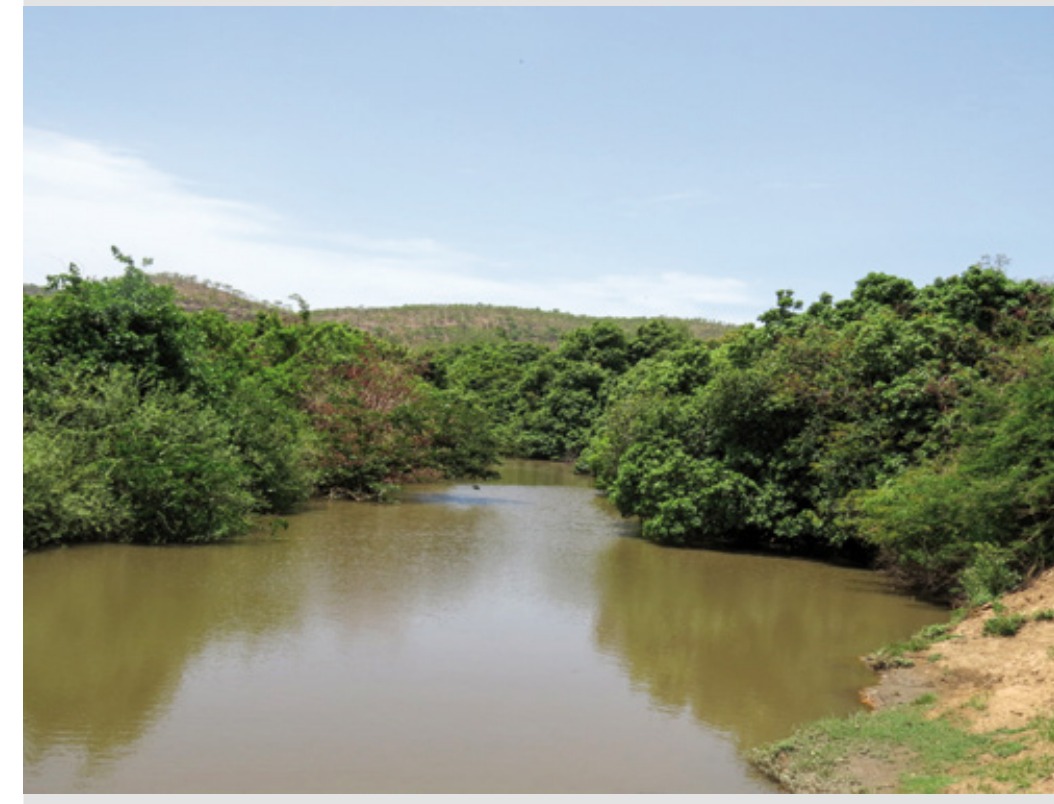

Photo 2.

Une forêt-galerie à Cola laurifolia le long de la rivière Pendjari.

Photo E. Assédé.

\section{Matériel et méthodes}

\section{Milieu d'étude}

L'étude a été conduite dans la Réserve de biosphère de la Pendjari (RBP) située à l'extrême nord-ouest de la République du Bénin entre $10^{\circ} 30$ et $11^{\circ} 30$ de latitude nord, $0^{\circ} 50$ et $2^{\circ} 00$ de longitude est. Située dans la zone phytogéographique soudanienne, la RBP comporte : le Parc national de la Pendjari (PNP) ou noyau central de la réserve d'une superficie de $2660 \mathrm{~km}^{2}$; la zone cynégétique de la Pendjari (ZCP) recouvrant $1750 \mathrm{~km}^{2}$; la zone cynégétique de Konkombri (ZCK) qui s'étend sur $251 \mathrm{~km}^{2}$ (figure 1). Ces trois zones sont entourées par les terroirs riverains. La RBP est relativement bien arrosée avec des précipitations annuelles moyennes de 1000 à $1100 \mathrm{~mm}$. La saison des pluies s'étend de mi-mai à octobre, et est suivie d'une saison sèche et fraîche marquée par l'harmattan, avec des minima de $12{ }^{\circ} \mathrm{C}$, allant de novembre à février, puis d'une saison sèche et chaude avec des maxima de $40^{\circ} \mathrm{C}$, courant de mars à mi-mai. Le paysage de la RBP recouvre plusieurs types de relief (plateaux, collines, montagne) associés à une diversité de sols. La végétation est une mosaïque de savanes (herbeuses, arbustives, arborées et boisées) et de forêts claires abritant une strate herbacée dominée par les graminées (photo 1). À ces formations bien réparties sur l'ensemble de la réserve s'ajoutent des galeries forestières bordant les cours d'eau (photo 2). 


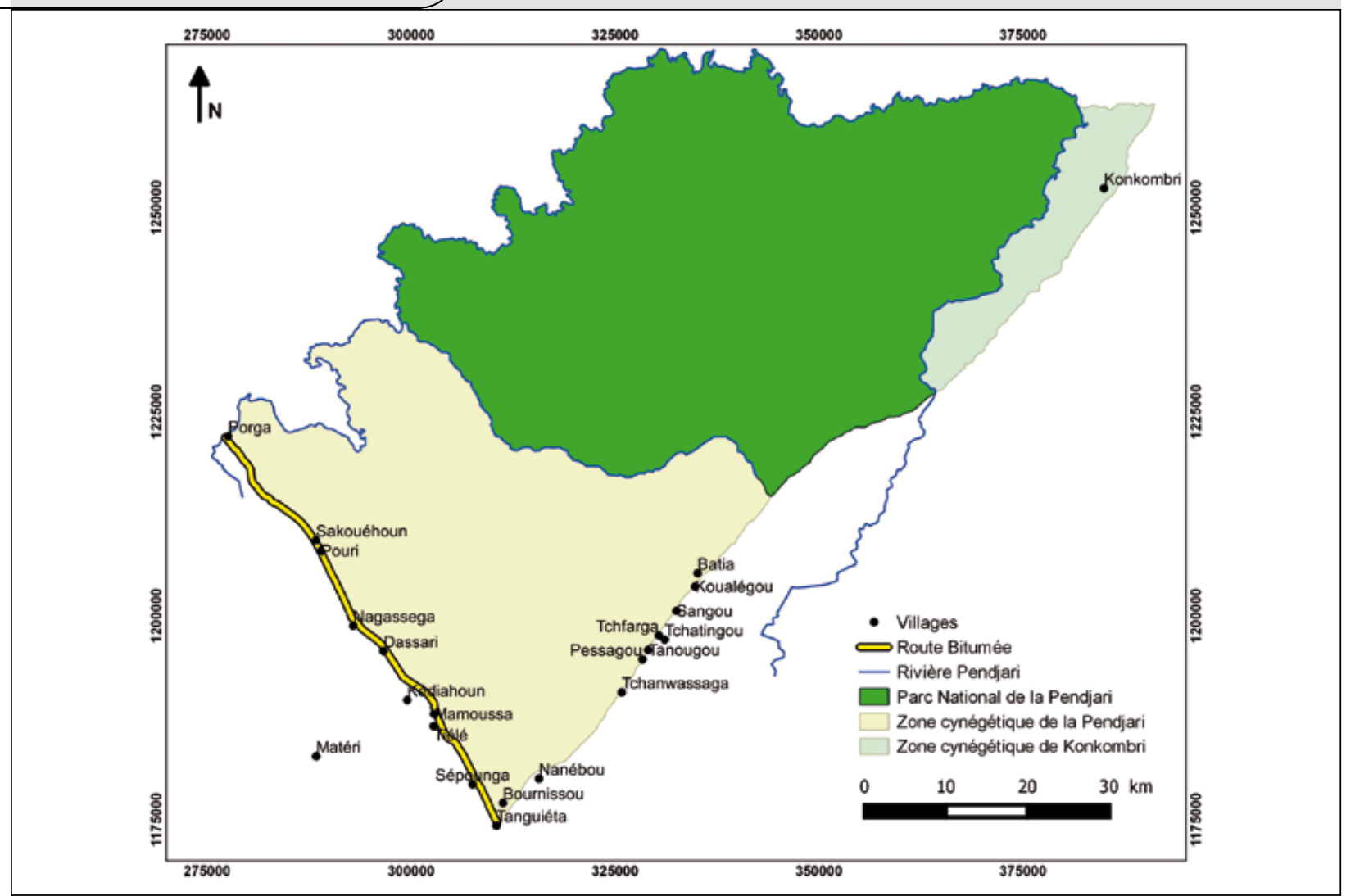

Figure 1.

Carte de la Réserve de biosphère de la Pendjari.

\section{Collecte des données}

L'inventaire de la régénération a été mené à la fin de la saison des pluies (septembre-octobre) à l'intérieur des 13 unités de végétation formant la végétation de la RBP (Assédé, 2014) :

- forêts claires à Anogeissus leiocarpa (DC.) Guill. \& Perr. sur plateaux de terre ferme à sols limono-argileux ;

- savanes arbustives à Combretum glutinosum Perr. ex DC. et Andropogon gayanus Kunth sur plateaux de terre ferme à sols ferrugineux gravillonnaires ;

- savanes arbustives et arborées à Crossopteryx febrifuga (G. Don) Benth sur plateaux de terre ferme, en buttes rocheuses à sols gravillonnaires ;

- savanes arbustives à Terminalia avicennioides Guill. \& Perr. et A. gayanus sur plateaux de terre ferme à sols sablo-argileux ; - savanes arborées à Vitellaria paradoxa C.F. Gaertn. ssp. paradoxa et $A$. gayanus sur collines à sols gravillonnaires ; - savanes arbustives et arborées à Burkea africana Hook. et Detarium microcarpum Guill. \& Perr. (photo 3) sur flancs des collines à sols rocailleux et gravillonnaires ;

- savanes arbustives à Acacia dudgeonii Craib ex Holland et A. gayanus sur plateaux de terre ferme à sols gravillonnaires; - forêts-galeries à Cola laurifolia Mast. sur bordures des cours d'eau à sols argilo-sableux ;

- forêts-galeries à Borassus aethiopum Mart. sur bordures des cours d'eau à sols sablo-limono-argileux ;
- savanes arborées à Terminalia macroptera Guill. \& Perr. et Mitragyna inermis (Willd.) Kuntze sur plaines alluviales à sols argilo-limoneux ;

- savanes arborées à M. inermis et Hyparrhenia glabriuscula (A. Rich.) Stapf sur plaines alluviales à sols limono-argileux; - savanes arborées à T. macroptera et Brachiaria jubata (Fig. \& De Not.) Stapf sur plaines alluviales à sols limono-argileux; - savanes arbustives à Acacia hockii De Wild. et Sorghastrum bipennatum (Hack.) Pilg. sur plaines alluviales à sols limono-argileux.

Au total, 123 placeaux de $900 \mathrm{~m}^{2}$ ont été installés à l'intérieur des treize unités de végétation, réparties dans les zones protégées de la RBP suivant la méthode de Braun-Blanquet (1964). Cinq placettes carrées de $25 \mathrm{~m}^{2}$ (photo 4) chacune ont été installées à l'intérieur des placeaux (une placette par angle et une placette au centre) pour un total de 615 placettes (tableau I). La régénération de toutes les espèces ligneuses à $1 \mathrm{~cm}$ de diamètre basal ou plus de $1 \mathrm{~m}$ de hauteur et moins de $5 \mathrm{~cm}$ de diamètre et/ ou moins de 1,5 $\mathrm{m}$ de hauteur a été dénombrée. En effet, l'exploration de terrain a montré que les tiges de diamètre basal supérieur à $1 \mathrm{~cm}$ sont celles qui ont survécu au passage des feux de végétation et à d'autres contraintes environnementales, et peuvent être recrutées au stade adulte. Toutes les régénérations (aussi bien issues de semis, rejets que de drageons) ont été considérées. Toutes les tiges ont été comptées pour les rejets à tiges multiples. 


\section{Analyse des données}

La densité de régénération a été calculée pour chaque placette. Une densité de régénération moyenne a été ensuite calculée par placeau, puis par unité de végétation. La densité de régénération totale a été calculée en considérant la densité de régénération des 13 unités de végétation étudiées. Le nombre total d'espèces en régénération et par unité de végétation a été déterminé.

La relation entre le relief et le potentiel de régénération des unités de végétation a été établie suivant la régression de Poisson. La loi de Poisson avec le modèle log-linéaire a permis de modéliser la densité de régénération et le nombre d'espèces en régénération suivant le type de relief. La variable indépendante est le relief. Deux formes de relief ont été considérées dans cette étude : les plateaux et les collines. S'agissant des plateaux, nous avons distingué trois variantes :

- les plaines à inondation temporaire et à hydromorphie élevée sur sols profonds, limoneux et limono-argileux ;

- les plateaux sur terre ferme sur sols variés ;

- les bordures de cours d'eau sur sols profonds argilo-sableux qui représentent ici les galeries forestières.

Quant aux collines, seuls les flancs des collines sur sols ferrugineux rocailleux ont été prises en compte comme une variante. Quatre variantes ou modalités du relief ont ainsi été considérées : plaine d'inondation, plateau, bordure des cours d'eau et colline. La densité de régénération des 123 placeaux échantillonnés a été organisée suivant les quatre modalités du relief. La modalité « plateau » est retenue comme le niveau de référence.

Deux modèles de régression ont été établis :

- modèle de Poisson suivant l'effet du relief sur la densité de régénération ;

- modèle de Poisson suivant l'effet du relief sur le nombre d'espèces en régénération (richesse spécifique).

Les coefficients de régression de Poisson et les taux d'incidence (IRR) ont été estimés pour chaque modèle (Long et Freese, 2005). L'erreur type, l'intervalle de confiance de $95 \%$ et la probabilité de la statistique de Fisher ont été calculés. Un test d'adéquation du modèle a été réalisé dans chaque cas en utilisant la déviance résiduelle. Le logiciel utilisé est R3.0.3 avec les « packages msm et sandwich ».

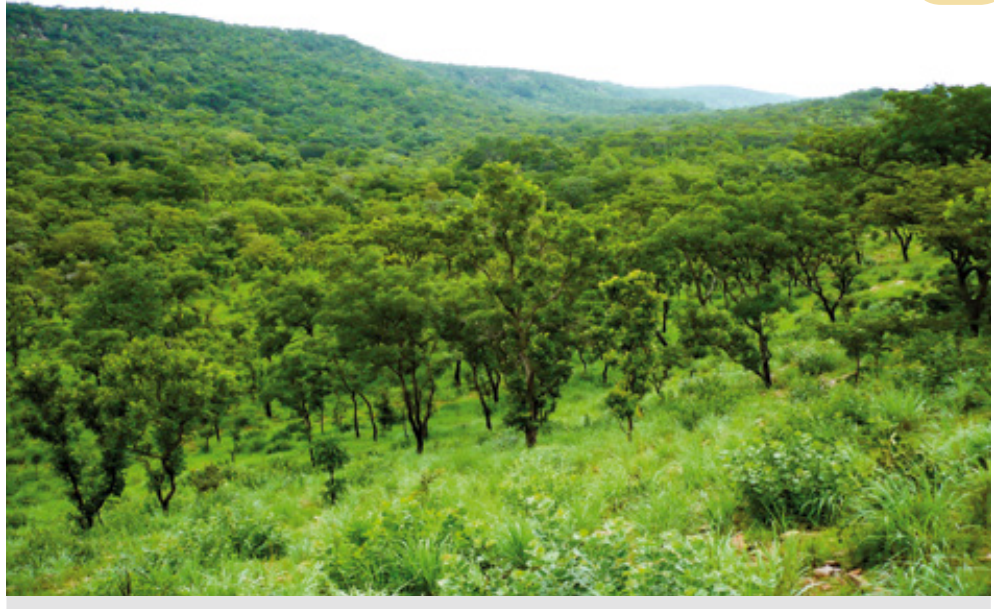

Photo 3.

Savanes arbustives et arborées à Burkea africana et Detarium microcarpum sur les flancs de colline. Photo E. Assédé.

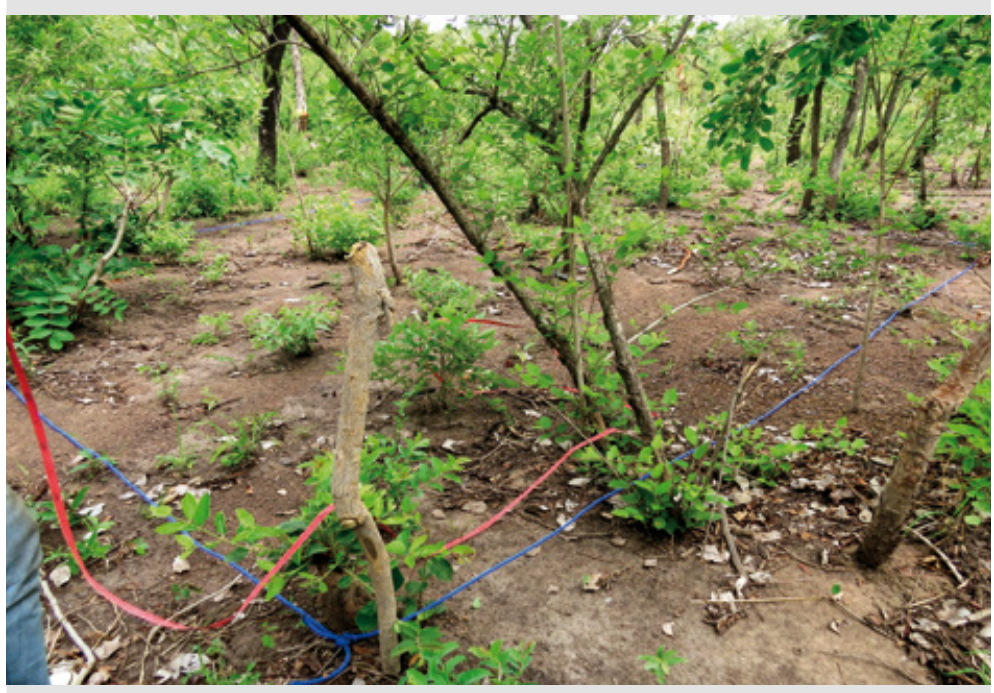

Photo 4.

Installation de placeau de $5 \mathrm{~m}^{2}$ sur plateau

pour l'inventaire de la régénération.

Photo E. Assédé.

\section{Tableau I.}

Nombre de placeaux et de placettes échantillonnés par unité de végétation.

\begin{tabular}{|c|c|c|c|c|c|c|c|c|c|c|c|c|c|c|}
\hline Unités de végétation & An & Co-An & Ter-An & Ac-An & Vi-An & $\mathrm{Cr}$ & $\mathrm{Bu}-\mathrm{De}$ & Co & Bo & Ter-Mi & Mi-Hy & Ter-Pa & So-Ac & Total \\
\hline Placeaux & 7 & 14 & 5 & 8 & 11 & 21 & 9 & 9 & 6 & 9 & 9 & 8 & 7 & 123 \\
\hline Placettes & 35 & 70 & 25 & 40 & 55 & 105 & 45 & 45 & 30 & 45 & 45 & 40 & 35 & 615 \\
\hline
\end{tabular}

Ter-Mi : savane arborée à Terminalia macroptera et Mitragyna inermis; Mi-Hy : savane arborée à Mitragyna inermis et Hyparrhenia glabriuscula ; Ter-Br : savane arborée à Terminalia macroptera et Brachiaria jubata; So-Ac : savane arbustive à Sorghastrum bipennatum et Acacia hockii ; Bo : forêt-galerie à Borassus aethiopum ; Co : forêt-galerie à Cola laurifolia ; An : forêt claire à Anogeissus leiocarpa ; Ter-An : savane arbustive à Terminalia avicennioides et Andropogon gayanus; Bu-De : savane arbustive et arborée à Burkea africana et Detarium microcarpum ; Ac-An : savane arbustive à Acacia dudgeonii et Andropogon gayanus; Co-An : savane arbustive à Combretum glutinosum et Andropogon gayanus; $\mathrm{Cr}$ : savane arbustive et arborée à Crossopteryx febrifuga ; Vi-An : savane arborée à Vitellaria paradoxa et Andropogon gayanus. 


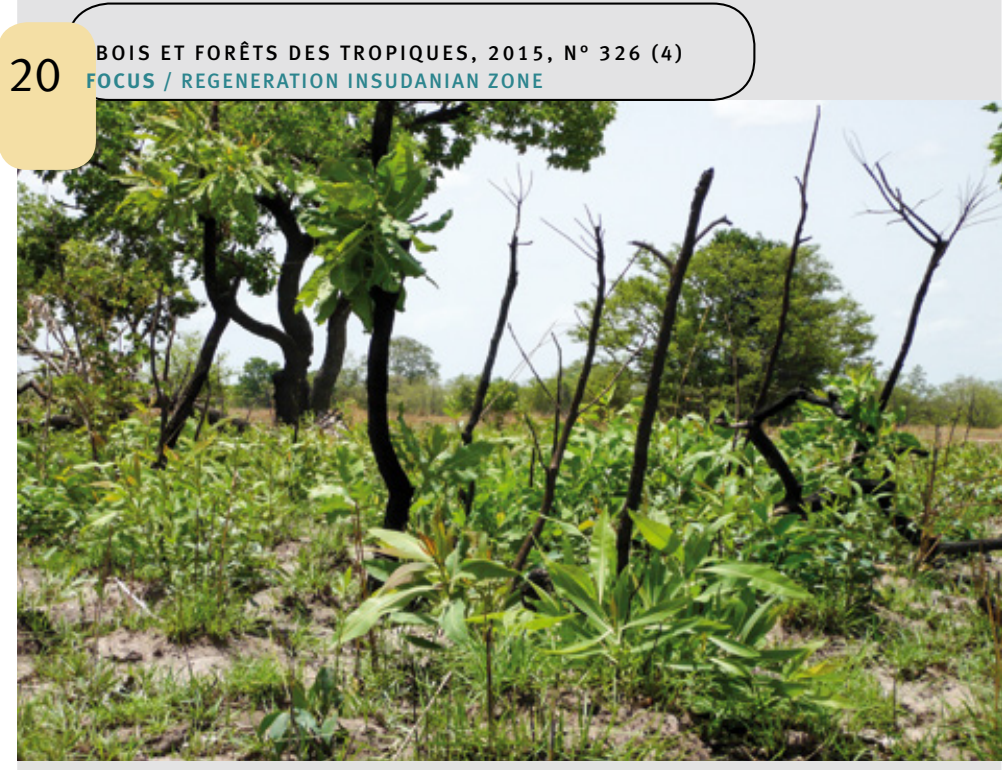

Photo 5.

Régénération de Terminalia macroptera

sur plaine d'inondation.

Photo E. Assédé.

\section{Résultats}

\section{Potentiel de régénération des unités de végétation}

La régénération des 13 unités de végétation étudiées est constituée de 73 espèces ligneuses réparties dans 56 genres et 24 familles. Les familles des Fabaceae, Combretaceae et Malvaceae sont les plus diversifiées avec respectivement $29 \%, 11 \%$ et $8 \%$ de la richesse floristique totale ; 54 $\%$ des familles recensées sont représentées par une seule espèce. Les trois espèces qui se régénèrent le plus dans la RBP sont $M$. inermis (7,6 \%), T. macroptera (5,1 \%) (photo 5) et $A$. dudgeonii ( $5 \%$ ).
La densité et la composition floristique de la régénération varient suivant les unités de végétation (tableau II) et le relief. La plus grande densité de régénération est observée sur les collines dans la savane arbustive et arborée à $B$. africana et $D$. microcarpum (photo 6). En revanche, la plus grande richesse spécifique est observée sur plateau en forêt claire à Anogeissus leiocarpa (DC.) Guill. et Perr. avec une nette dominance des régénérations de $A$. leiocarpa. Dans les savanes arbustives à $T$. avicennioides et $A$. gayanus installées sur plateau, seules $T$. avicennioides et Pterocarpus erinaceus Poir. impriment leur dominance dans la régénération.

\section{Relation entre le potentiel de régénération et le relief}

La relation entre le potentiel de régénération (densité de régénération et richesse spécifique) et le relief est résumée dans les tableaux III et IV. Soit $\lambda_{\text {plateau }}, \lambda_{b}, \lambda_{c}$ et $\lambda_{p}$ les densités de régénération observées respectivement sur plateau, bordure de cours d'eau, colline et plaine d'inondation. Soit $\beta_{\text {plateau }}$, $\beta_{b}$, $\beta_{c}$ et $\beta_{p}$ les nombres d'espèces en régénération observés respectivement sur les plateaux, bordures de cours d'eau, collines et plaines d'inondation.

\section{Effet du relief sur la densité de régénération}

Comparativement au niveau de référence « plateau », les bordures de cours d'eau et les collines ont un effet positif sur la densité de régénération, tandis que les plaines d'inondation ont un effet négatif (tableau III). Les densités de régénération observées sur ces trois variantes du relief sont respectivement de 1,01, 2,10 et 0,67 fois celle observée sur plateaux (tableau IV). La densité de régénération des ligneux augmente des plateaux vers les bordures de cours d'eau $\left(\lambda_{b}\right)$ et collines $\left(\lambda_{c}\right)$, mais diminue des plateaux vers les plaines d'inondation $\left(\lambda_{p}\right)$ :

Tableau II.

Caractéristique floristique et densité moyenne de régénération des unités de végétation.

$\begin{array}{ll}\text { Unité de végétation } & \text { Topographie }\end{array}$

Forêt claire à Anogeissus leiocarpa

Plateau

Richesse Densité régénération spécifique (tiges/ha)

\begin{tabular}{|c|c|c|c|}
\hline Savane arbustive à Combretum glutinosum et Andropogon gayanus & Plateau & 45 & $8691,4 \pm 229$ \\
\hline Savane arbustive à Terminalia avicennioides et Andropogon gayanus & Plateau & 22 & $3840,1 \pm 152,6$ \\
\hline Savane arborée à Vitellaria paradoxa et Andropogon gayanus & Plateau & 35 & $3527,2 \pm 111,5$ \\
\hline Savane arbustive et arborée à Burkea africana et Detarium microcarpum & Colline & 32 & $18640,1 \pm 683$ \\
\hline Savane arbustive et arborée à Crossopteryx febrifuga & Colline & 36 & $15887,6 \pm 569$ \\
\hline Savane arbustive à Acacia dudgeonii et Andropogon gayanus & Plaine d'inondation & 23 & $9930,1 \pm 673,6$ \\
\hline Forêt-galerie à Cola laurifolia & Bordure de cours d'eau & 18 & $7902,2 \pm 588$ \\
\hline Forêt-galerie à Borassus aethiopum & Bordure de cours d'eau & 19 & $8628,6 \pm 597$ \\
\hline Savane arborée à Terminalia macroptera et Mitragyna inermis & Plaine d'inondation & 26 & $7200,4 \pm 612$ \\
\hline Savane arborée à Mitragyna inermis et Hyparrhenia glabriuscula & Plaine d’inondation & 21 & $9057,7 \pm 1030$ \\
\hline Savane arborée à Terminalia macroptera et Brachiaria jubata & Plaine d'inondation & 22 & $2080,1 \pm 927,8$ \\
\hline Savane arbustive à Sorghastrum bipennatum et Acacia hockii & Plaine d’inondation & 10 & $1714,3 \pm 242,6$ \\
\hline
\end{tabular}


$\lambda_{b}=1,01 \lambda_{\text {plateau }}$

$\lambda_{c}=2,1 \lambda_{\text {plateau }}$

$\lambda_{p}{ }^{c}=0,67 \lambda_{\text {plateau }}$

Effets du relief sur la richesse spécifique

Comparativement au niveau de référence " plateau ", toutes les autres variantes du relief en dehors des collines ont un effet négatif sur le nombre d'espèces en régénération (tableau III). Les nombres d'espèces en régénération sur les plaines d'inondation et les bordures de cours d'eau sont res- pectivement de 0,43 et 0,6 fois celui observé sur plateau. En revanche, le nombre d'espèces en régénération sur les collines est de 1,2 fois celui observé sur plateau (tableau IV). Le nombre d'espèces en régénération augmente des plateaux $\left(\beta_{\text {plateau }}\right)$ vers les collines $\left(\beta_{c}\right)$, mais diminue des plateaux vers les plaines d'inondation $\left(\beta_{p}\right)$ et les bordures de cours d'eau $\left(\beta_{b}\right)$ :

$$
\begin{aligned}
& \beta_{b}=0,6 \beta_{\text {plateau }} \\
& \beta_{c}=1,2 \beta_{\text {plateau }} \\
& \beta_{p}=0,43 \beta_{\text {plateau }}
\end{aligned}
$$

\begin{tabular}{|c|c|c|c|c|}
\hline & Coef & Erreur type & $\mathbf{z}$ & $\operatorname{Pr}(>|z|)$ \\
\hline $\begin{array}{l}\text { Densité de régénération } \\
\text { Constante }\end{array}$ & 8,997 & 0,001 & 5303,28 & $<2 \mathrm{e}-16$ \\
\hline Colline & 0,728 & 0,002 & 104,74 & $<2 \mathrm{e}-16$ \\
\hline Bordure de cours d'eau & 0,013 & 0,003 & 4,14 & $3,36 e-5$ \\
\hline Plaine d'inondation & $-0,39$ & 0,003 & $-133,23$ & $<2 \mathrm{e}-16$ \\
\hline $\begin{array}{l}\text { Richesse spécifique } \\
\text { Constante }\end{array}$ & 2,492 & 0,043 & 56,84 & $<2 \mathrm{e}-16$ \\
\hline Colline & 0,192 & 0,064 & 2,98 & 2,89e-03 \\
\hline Bordure de cours d'eau & $-0,537$ & 0,106 & $-5,041$ & $4,63 e-07$ \\
\hline Plaine d’inondation & $-0,834$ & 0,088 & $-9,4$ & $<2 \mathrm{e}-16$ \\
\hline
\end{tabular}

Tableau III.

Coefficients de régression de Poisson liés à la densité de régénération et à la richesse spécifique.

\section{Tableau IV.}

\begin{tabular}{|c|c|c|c|c|}
\hline & IRR & Erreur type & [95 \% IC & $95 \%$ IC] \\
\hline $\begin{array}{l}\text { Densité de régénération } \\
\text { Constante }\end{array}$ & 8080 & 1,111 & 6577,19 & 9926,184 \\
\hline Colline & 2,071 & 1,13 & 1,63 & 2,631 \\
\hline Bordure de cours d'eau & 1,014 & 1,19 & 0,721 & 1,425 \\
\hline Plaine d'inondation & 0,677 & 1,215 & 0,462 & 0,991 \\
\hline $\begin{array}{l}\text { Richesse spécifique } \\
\text { Constante }\end{array}$ & 12,093 & 1,04 & 11,035 & 13,252 \\
\hline Colline & 1,213 & 1,056 & 1,089 & 1,350 \\
\hline Bordure de cours d'eau & 0,584 & 1,089 & 0,493 & 0,691 \\
\hline Plaine d'inondation & 0,434 & 1,104 & 0,357 & 0,527 \\
\hline
\end{tabular}

Taux d'incidence des coefficients de régression de Poisson liés à la densité de régénération et à la richesse spécifique. 
Tableau V.

Test d'ajustement du modèle de Poisson.

\begin{tabular}{|c|c|c|c|c|}
\hline Variable explicative & Variable expliquée & Déviance résiduelle & df & $\mathbf{p}$ \\
\hline Topographie & Régénération spécifique & 114,55 & 116 & $0,52 \mathrm{~ns}$ \\
\hline Topographie & Régénération & 383698 & 116 & $0,55 \mathrm{~ns}$ \\
\hline
\end{tabular}

\section{Signification des modèles}

Le test d'adéquation des deux modèles (modèle de Poisson suivant l'effet du relief sur la densité de régénération et modèle de Poisson suivant l'effet du relief sur le nombre d'espèces en régénération) effectué sur les déviances résiduelles n'est pas significatif dans les deux cas (respectivement $P=0,1$ et $P=0,5)$. Nous concluons donc que les deux modèles s'ajustent et que la variable relief est un prédicteur statistiquement significatif de la densité de régénération et de la richesse spécifique (tableau V).

\section{Discussion}

\section{Potentiel de régénération des unités de végétation}

Le potentiel de régénération des unités de végétation en zone soudanienne reflète bien la composition de la végétation en place. La famille des Fabaceae, dominante dans la haute strate (Assédé et al., 2012), s’impose également dans la régénération. L'importance de la composante adulte de cette famille dans la RBP pourrait expliquer la dominance de ses espèces dans la régénération. Cela s'expliquerait par les stratégies de reproduction et de survie des espèces de cette famille. Les Fabaceae se reproduisent bien dans des milieux secs aux plus humides (Tybirk, 1997 ; Ouédraogo et al., 2006). Le transport des fruits des Papillionaceae et Mimosaceae par le vent (anémochorie) favoriserait une large dispersion des graines (Tybirk, 1997). La persistance du stock en graines des Fabaceae dans le sol permet également à la régénération de s'exprimer dans la période favorable. Cependant, l'utilisation du feu comme principal outil d'aménagement des unités de végétation dans le domaine soudanien (Scott, 2000 ; Traoré et al., 2008) en général et dans la Réserve de Biosphère de la Pendjari en particulier serait le premier facteur stimulateur de la régénération des Fabaceae et de certaines Combretaceae. L'effet additif du choc thermique et de la fumée dégagée lors de la combustion de la végétation sur la levée de la dormance des Fabaceae a été largement démontré (Dayamba et al., 2008 ; Niang-Diop et al., 2010 ; Holou et al., 2011). Pour certaines légumineuses tropicales, les températures comprises entre 80 et $100{ }^{\circ} \mathrm{C}$ font augmenter d'une manière significative la germination des graines. Pour d'autres scientifiques, cette germination est stimulée par la fumée produite par la combustion de la végétation (Razanamandranto et al., 2005 ; Daws et al., 2007). L'existence d'une relation de symbiose entre les racines des Fabaceae et des bactéries (Rhizobium) et champignons capables de fixer l'azote atmosphérique stimule également la régénération, même sur des sols pauvres en azote (Raddad et al., 2005 ; Kuyper et al., 2004). De nombreuses espèces de Caesalpiniaceae auraient ainsi co-évolué avec les champignons ectomycorhiziens ou mycorhiziens à arbuscules et vésicules en gage d'adaptation à des habitats spécialisés (Fitter et Moyersoen, 1996 ; Kuyper et al., 2004). C'est le cas de Detarium microcarpum qui forme des associations mycorhiziennes à arbuscules et vésicules favorisant ainsi son établissement sur des sols pauvres en azote. Toutefois, cet effet stimulateur de la régénération des Fabaceae n'est pas reconnu par tous (Danthu et al., 2003).

\section{Relation entre topographie et potentiel de régénération}

La variable relief semble bien prédire la variation spatiale de la densité de régénération et le nombre d'espèces en régénération. Contrairement aux collines et aux bordures de cours d'eau, les plaines d'inondation se présenteraient comme des milieux très spécialisés où peu d'espèces peuvent exprimer leur potentiel de régénération. Il s'agit de milieux en forme de cuvette avec un niveau plus bas que celui des bordures des cours d'eau. Ceci justifie qu'en période de

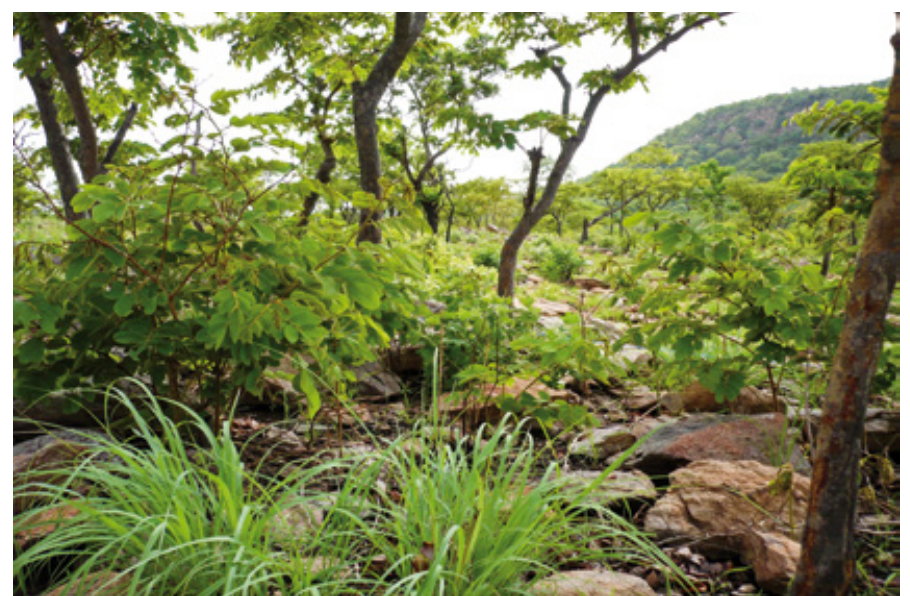

Photo 6.

Régénération de Detarium microcarpum sur flanc de colline. Photo E. Assédé. 
crue, l'eau stagne davantage dans ces plaines d'inondation qu'en bordure des cours d'eau. Le niveau d'asphyxie assez élevé des plaines d'inondation exige des stratégies de survie particulières des espèces. En outre, le battement des eaux pendant les périodes de crue et de décrue, caractéristique de ces plaines d'inondation, ne permet pas aux jeunes plantules de nombreuses espèces ligneuses après germination de s'établir sur ce milieu de vie. Ce qui explique en partie la réduction aussi bien de la densité de régénération que du nombre d'espèces en régénération des plateaux vers les plaines d'inondation. Dans la même logique, Bondé et al. (2013) avaient montré que les formations des plaines d'inondation étaient moins diversifiées que celles des plateaux car les écosystèmes de plateaux sont peu sélectifs et offrent des conditions édaphiques favorables à l'installation et au développement de plusieurs espèces.

Cependant, même si les données s'ajustent aux deux modèles de Poisson, la probabilité du test de qualité de l'ajustement est assez faible pour deux raisons. La première raison, les variables prédictives utilisées dans le modèle seraient insuffisantes (Long, 1997 ; Long et Freese, 2005). Autrement dit, il existerait des variables prédictives omises car, même si le relief est un bon prédicteur du potentiel de régénération, celui-ci n'en est pas le seul déterminant. De nombreux autres facteurs tels que les propriétés physico-chimiques et biologiques du sol (matière organique, azote, phosphore, texture, etc.) et l'insolation sont également déterminants dans l'établissement du potentiel de régénération. Biaou et al. (2011) ont démontré l'importance de l'insolation dans la survie de la régénération. La seconde raison possible est l'existence d'une surdispersion des données (Mc Cullagh et Nelder, 1989). L'hétérogénéité au sein des placeaux, non prise en compte par les modèles, pourrait expliquer cette dispersion. En effet, selon Basnet (1990) et Whitmore (1978), les caractéristiques topographiques sont des facteurs physiques à la base de la diversité des microsites, qui varient eux-mêmes selon le type de sol, les régimes d'humidité, la formation géologique. Ces microsites déterminent en retour la répartition des espèces.

\section{Conclusion}

Le potentiel de régénération des unités de végétation de la RBP varie avec le relief. Ce potentiel de régénération, qui reflète bien la structure de la végétation en place, est beaucoup plus faible dans les plaines d'inondation et les bordures de cours d'eau, si l'on se réfère aux plateaux. Cette étude fait ressortir la nécessité d'un suivi à long terme de la régénération, surtout dans les plaines d'inondation et les galeries forestières, afin de mieux comprendre la dynamique de la régénération. Une stratégie de conservation des plaines d'inondation doit être développée dans le plan d'aménagement de la RBP, y compris dans une perspective de mise en défens. Les feux de brousse étant le principal facteur perturbateur des espèces végétales dans la RBP, des études sur l'impact du feu sur la survie de la régénération suivant le relief permettront de mieux comprendre cette variation du potentiel de régénération.

\section{Remerciements}

Les auteurs remercient le Bundesministerium für Bildung und Forschung (BMBF) pour le financement de cette étude à travers le projet BIOTA-Ouest III. Les auteurs expriment également leur gratitude à B. Fandohan et M. D. Kouton pour leurs commentaires et aux populations locales autour de la Réserve de biosphère de la Pendjari pour leur collaboration pendant la collecte des données sur le terrain, ainsi qu'aux personnes ayant contribué à la publication de ce manuscrit.

\section{Références bibliographiques}

Assédé E. P. S., 2014. Ecology of plant communities in the Biosphere Reserve of Pendjari, Benin (West Africa). Ph.D. Dissertation, Abomey-Calavi University, Bénin, 360 p.

Assédé E. P. S., Adomou A. C., Sinsin B., 2012. Magnoliophyta, Biosphere Reserve of Pendjari, Atacora Province, Benin. Check list, 8 (4): 642-661.

Avocevou-Ayisso C., Sinsin B., Adégbidi A., Dossou G., Van Damme P., 2009. Sustainable use of non-timber forest products: Impact of fruit harvesting on Pentadesma butyracea regeneration and financial analysis of its products trade in Benin. Forest Ecology and Management, 257: 1930-1938.

Badji M., Sanogo D., Apko L. E., 2014. Dynamique de la végétation ligneuse des espaces sylvopastoraux villageois mis en défens dans le Sud du Bassin arachidier au Sénégal. Bois et Forêts des Tropiques, 319 (1) : 43-52. [En ligne] http://bft.cirad.fr/cd/BFT 319 43-52.pdf

Basnet K., 1990. Studies of ecological and geological factors controlling the pattern of tabonuco forest in the Luquillo Experimental Forest, Puerto Rico. Ph.D. Dissertation, Rutgers University, USA.

Biaou S. S. H., Holmgren M., Sterck F. J., Mohren G. M. J., 2011. Stress-Driven Changes in the Strength of Facilitation on Tree Seedling Establishment in West African Woodlands. Biotropica, 43 (1): 23-30.

Bondé L., Ouédraogo O., Kagambega F., Boussim J. I., 2013. Impact des gradients topographique et anthropique sur la diversité des formations ligneuses soudaniennes. Bois et Forêts des Tropiques, 318 (4) : 15-26. [En ligne] http://bft. cirad.fr/cd/BFT 318 15-25.pdf

Braun-Blanquet J., 1964. Pflanzensoziologie. Grundzüge der Vegetationskunde. $3^{\text {e }}$ éd. Vienne, Autriche, New York, USA, Springer, $865 \mathrm{p}$.

Braun-Blanquet J., 1979. Fitosociologia. Bases para el estudio de las comunidades vegetales, Madrid, Espagne, Ed. Blume.

Braun-Blanquet J., Roussine N., Nègre R., 1952. Les groupements végétaux de la France méditerranéenne. Paris, France, CNRS, 292 p.

Bucci G., Borghetti M., 1997. Understory vegetation as a useful predictor of natural regeneration and canopy dynamics in Pinus sylvestris forests in Italy. Acta Ecologica, 18 (4): 485-501.

Coop J. D., Massatti R. T., Schoettle A. W., 2010. Subalpine vegetation pattern three decades after stand-replacing fire: effects of landscape context and topography on plant community composition, tree regeneration, and diversity. Journal of Vegetation Science, 21 (3): 472-487. 
Danthu P., Ndongo M., Diaou M., Thiam O., Sarr A., Dedhiou B., Vall O. M., 2003. Impact of bush fire on germination of some West African acacias. Forest Ecology and Management, 173: 1-10.

Daws M. I., Davies J., Pritchard H. W., Brown N. A. C., Van-Staden J., 2007. Butenolide from plant-derived smoke enhances germination and seedling growth of arable weed species. Plant Growth Regulation, 51: 73-82.

Dayamba S. D., Tigabu M., Sawadogo L., Oden P. C., 2008. Seed germination of herbaceous and woody species of the Sudanian savanna-woodland in response to heat shock and smoke. Forest Ecology and Management, 256: 462-470.

Fitter A. H., Moyersoen B., 1996. Evolutionary trends in root-mycrobe symbioses. Philosophical Transactions of the Royal Society B, 351: 1367-1375.

Gaoué O. G., Ticktin T., 2007. Patterns of harvesting foliage and bark from the multipurpose tree Khaya senegalensis in Benin: Variation across ecological regions and its impacts on population structure. Biological Conservation, 137: 424-436.

Gaoué O. G., Ticktin T., 2009. Effects of Harvest of Non timber Forest Products and Ecological Differences between Sites on the Demography of African Mahogany. Conservation Biology, 24 (2) : 605-614.

Holou R., Teka O., Kindomihou V., Sinsin B., 2011. Germination et utilisation de Caesalpinia benthamiana (Baillon) P.S. Herendeen \& J.L. Zarucchi (Leguminosae-Caesalpiniaceae) dans l'aménagement anti-érosif des retenues d'hydraulique pastorale au Bénin. International Journal of Biological and Chemical Sciences, 5 (2): 533-545.

Koulibaly A., Kouamé N. F., Traoré D., Porembski S., 2010. Structure et régénération de la végétation ligneuse, le long de transects forêts-savanes, dans la région de la réserve de Lamto (Côte d'Ivoire). Annales de Botanique de l'Afrique de l'Ouest, $6:$ :56-72.

Kuyper T. W., Cardoso I. M., Onguene N. A., Murniati, van Nordwijk M., 2004. Managing mycorrhiza in tropical multispecies agroecosystems. In: van Nordwijk M., Cadisch G., Ong C. K. (eds). Below-ground interactions in tropical agroecosystems. Concepts and models with multiple plant components. Wallingford, Royaume-Uni, CAB International, 243-261.

Ky-Dembele C., Tigadu M., Bayala J., Ouédraogo S. J., Oden P. C., 2007. The relative importance of different regeneration mechanisms in a selectively cut savanna-woodland in Burkina Faso, West Africa. Forest Ecology and Management, 243: 28-38.

Long J. S., 1997. Regression Models for Categorical and Limited Dependent Variables. Londres, Royaume-Uni, SAGE, 328 p.

Long J. S., Freese J., 2005. Regression Models for Categorical Dependent Variables Using Stata. Second edition. College Station, TX, USA, Stata Press, 527 p.

Maingi J. K., Marsh E. E., 2006. Composition, structure, and regeneration patterns in a gallery forest along the Tana River near Bura, Kenya. Forest Ecology and Management, 236: 211-228.

Mama A., Bamba I., Sinsin B., Bogaert J., De Cannière C., 2014. Déforestation, savanisation et développement agricole des paysages de savanes-forêts dans la zone soudano-guinéenne du Bénin. Bois et Forêts des Tropiques, 324 (4) : 65-75. [En ligne] http://bft.cirad.fr/cd/BFT 322 65-75.pdf
Mc Cullagh P., Nelder J. A., 1989. Generalized Linear Models. Londres, Royaume-Uni, Chapman and Hall/CRC, 532 p.

Nacoulma B. M. I., Traoré S., Hahn K., Thiombiano A., 2011. Impact of land use types on population structure and extent of bark and foliage harvest of Afzelia africana and Pterocarpus erinaceus in Eastern Burkina Faso. International Journal of Biodiversity and Conservation, 3 (3): 62-72.

Niang-Diop F., Sambou B., Lykke A. M., 2010. Contraintes de régénération naturelle de Prosopis africana : facteurs affectant la germination des graines. International Journal of Biological and Chemical Sciences, 4 (5): 1693-1705.

Ouédraogo A., Thiombiano A., Hahn-Hadjali K., Guinko S., 2006. Structure du peuplement juvénile et potentialités de régénération des ligneux dans l'Est du Burkina Faso. Études de la Flore et de la Végétation du Burkina Faso, 10 :17-24.

Ouédraogo A., Thiombiano A., Hahn-Hadjali K., Guinko S., 2009. Diversité et dynamique de la végétation ligneuse juvénile du Parc national d’Arly (Burkina Faso). Candollea, 64: 257-278.

Raddad E. Y., Salih A. A., Elfadl M. A., Kaarakka V., Luukkanen O., 2005. Symbiotic nitrogen fixation in eight Acacia senegal provenances in dryland clays of the Blue Nile Sudan estimated by the ${ }^{15} \mathrm{~N}$ natural abundance method. Plant and Soil, 275 (1): 261-269.

Razanamandranto S., Tigadu M., Sawadogo L., Oden P. C., 2005. Seed germination of eight savanna-woodland species from West Africa in response to different cold smoke treatments. Seed Science and Technology, 33: 315-328.

Robert A., 2003. Simulation of the effect of topography and tree falls on stand dynamics and stand structure of tropical forests. Ecological Modelling, 167: 287-303.

Schumann K., Wittig R., Thiombiano A., Becker U., Hahn K., 2011. Impact of land-use type and harvesting on population structure of a non-timber forest product-providing tree in a semi-arid savanna, West Africa. Biological Conservation, 144: 2369-2376.

Scott A. C., 2000. The Pre-Quaternary history of fire. Palaeogeography, Palaeoclimatology, Palaeoecology, 164: 281-329.

Sinsin B., Eyog-Matig O., Assogbadjo A. E., Gaoué O. G., Sinadouwirou T., 2004. Dendrometric characteristics as indicators of pressure of Afzelia africana Sm. trees dynamics in different climatic zones of Benin. Biodiversity and Conservation, 13 (8): 1555-1570.

Traoré S., Kaboré O., Rasolodimby J. M., Thiombiano L., Guinko S., 2008. Impact of Protected Areas and Land Use on Regeneration of Acacia Woodland's in Eastern Burkina Faso. Flora et Vegetatio Sudano-Sambesica, 11: 17-24.

Tybirk K., 1997. Reproductive biology and evolution of the genus Acacia. Bulletin of the International Group for the Study of Mimosoideae, 20: 45-53.

Whitmore T. C., 1978. Gaps in the forest canopy. In: Tomlinson P. B., Zimmermann M. H. (eds). Tropical tree as living systems. Cambridge, Royaume-Uni, Cambridge University Press, 639-655. 